\title{
Amplitude modulation on millimeter-wave signal with low driving voltage using reciprocating optical modulator
}

\author{
Takahide Sakamoto ${ }^{\text {a)}}$, Tetsuya Kawanishi, Satoshi Shinada, \\ and Masayuki Izutsu \\ National Institute of Information and Communications Technology \\ 4-2-1 Nukui-kitamachi, Koganei-shi, Tokyo 184-8795, Japan \\ a) tsaka@nict.go.jp
}

\begin{abstract}
A reciprocating optical modulator (ROM) generates a millimeter-wave signal in good efficiency. Internal bias modulation on a ROM is reported, in this paper. Owing to reciprocating modulation in the ROM, the driving voltage required for amplitude modulation on a millimeter-wave signal is highly decreased. In this paper, a millimeterwave signal in the $52.8-\mathrm{GHz}$ frequency band is amplitude-modulated with a low-voltage $(2.0 \mathrm{~V})$ baseband signal, where half-wave voltage of the modulator embedded in the ROM is $6.4 \mathrm{~V}$.
\end{abstract}

Keywords: reciprocating optical modulator, millimeter-wave generation, radio-on-fiber system, amplitude-shift keying

Classification: Photonics devices, circuits, and systems

\section{References}

[1] W. D. Jemison, "Fiber radio: from links to networks," Microwave Photonics 2001, pp. 169-172, 2001.

[2] K. S. Abedin et al., "Higher order FM mode locking for pulse-repetition enhancement in actively mode-locked lasers: theory and experiment," IEEE J. Quantum. Electron., vol. 35. pp. 875-890, 1999.

[3] K. Noguchi et al., "Millimeter-wave Ti:LiNbO 3 optical modulators," J. Lightwave Technol., vol. 16, pp. 615-619, 1998.

[4] T. Kawanishi et al., "Reciprocating optical modulation for harmonic generation," IEEE Photon. Technol. Lett., vol. 13. pp. 854-856, 2001.

\section{Introduction}

One of the most important challenges in radio-on-fiber (ROF) systems is to develop photonic millimeter-wave generators and up-converters with simple methods [1]. Many techniques for millimeter-wave generation have been proposed, so far. A mode-locked laser is a typical one [2] but has a problem in 
stability and highly suffers from phase noise. Moreover, an additional external modulator is indispensable to up-conversion of a baseband signal into a radio-on-fiber signal. To use a wideband optical modulator [3] is an alternative way but its modulation frequency and conversion efficiency is limited.

Contrast to these techniques, a reciprocating optical modulator ( $\mathrm{ROM}$ ) proposed in a previous report generates millimeter-wave signal in high stability and in good efficiency [4]. Another merit of this technique is that a millimeter wave is generated in low cost without high-frequency RF circuits, since the signals fed to the ROM are multiplied in frequency many times.

In this paper, we experimentally demonstrate amplitude modulation on a millimeter-wave signal using the ROM. Focusing on the optically multiplying process in the ROM, we propose that the millimeter-wave modulated lightwave at the output of the ROM is modulated in amplitude, which is realized by adding a baseband signal on a bias voltage introduced into the ROM. Therein, neither external modulator nor up-converter is required. By the internal bias-modulation technique, a $52.8-\mathrm{GHz}$ ROF signal is amplitude modulated with 2-Mbit/s binary data.

\section{Principles}

Basic structure of the ROM is shown in Fig. 1 (a). An optical phase modulator is sandwiched with twin band-reflection filters like fiber bragg gratings (FBGs). The mechanism of millimeter-wave generation with a ROM is explained as follows: A lightwave is injected into the ROM through the input filter, where the wavelength of the light is out of the reflection band of the filter. The light is phase-modulated by RF signals fed to the modulator and a part of its sideband components is reflected back to the modulator section, while the rest of them pass through the output filter. The reflected lightwave components are trapped inside the ROM cavity as long as their wavelengths coincide with the reflection band of the filters. Each time introduced into the modulator section, higher-order sideband is generated one after another. The higher-order sideband components out of the reflection band of the output filter are taken out from the output port. By detecting the optical beat between the lightwaves appeared both sides of the stop band of the output filter, millimeter wave is generated.

In such reciprocating modulation processes, the lightwaves traveling inside the cavity interfere with each other. The amplitude of the generated millimeter wave is maximized at the constructive interferometric condition, while minimized at the destructive case. A bias voltage applied on the phase modulator in the ROM cavity easily controls the interferometric conditions. Therefore, amplitude modulation on the millimeter wave is achieved by introducing a baseband signal into the bias port of the ROM together with the RF signal, as shown in Fig. 1(b). This scheme for amplitude-modulated millimeter-wave generation is so simple in the sense that no additional external modulator or up-converter is required. 
(a)

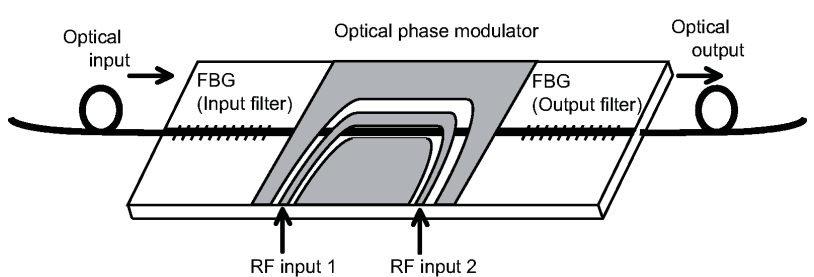

(b)
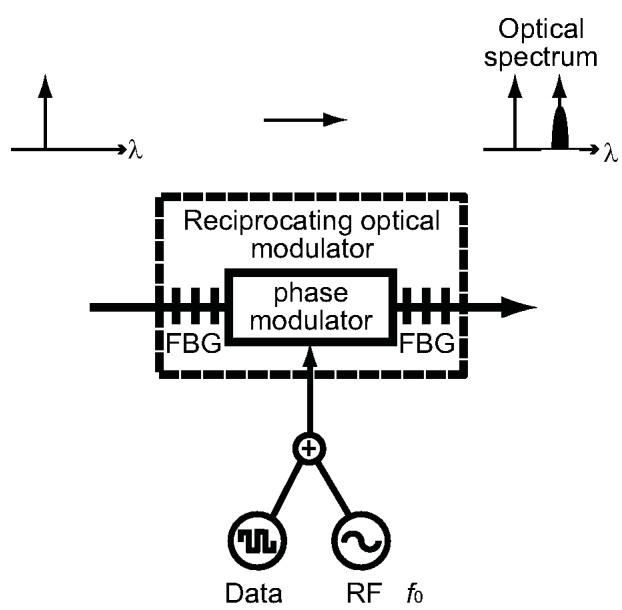

Fig. 1. (a) Basic structure of a hybrid-integrated reciprocating optical modulator (ROM).

(b) Principle of ASK-ROF generation based on the internal bias modulation on a ROM.

\section{Amplitude modulation on millimeter-wave signal with low driving voltage}

Fig. 2 shows the experimental setup for amplitude modulation on a millimeter wave signal generated using a ROM. The ROM consisted of a phase modulator and a pair of FBGs. The phase modulator, made of a $\mathrm{LiNbO}_{3}$ waveguide and a traveling-wave electrode, had a half-wave voltage of $6.4 \mathrm{~V}$. The FBGs were butt-jointed on both facets of the LN modulator and had the following same characteristics: the center wavelength was $1552 \mathrm{~nm}$ and the stop bandwidth was $0.4 \mathrm{~nm}$, respectively. This hybrid integration highly stabilizes the optical path in the ROM cavity, which is very effective to remove interferometric instability in the reciprocating modulation processes [4].

A CW light launched on the ROM was generated by a tunable laser diode. The input power was $0.0 \mathrm{dBm}$ and the center wavelength was aligned at $1551.8 \mathrm{~nm}$, which was just near by the FBG stop band. A 4.4-GHz sinusoidal RF signal was introduced into the ROM through the RF feeding port (a) shown in Fig. 2. The RF signal was divided half by an $180^{\circ}$-hybrid coupler and they were injected into the phase modulator section of the ROM in opposite directions through port (c) and (d), respectively. The feeder cables were aligned at the same length to obtain synchronous in-phase modulation during the round trips in the ROM.

DC bias and baseband signal voltages were applied on the bias input port 


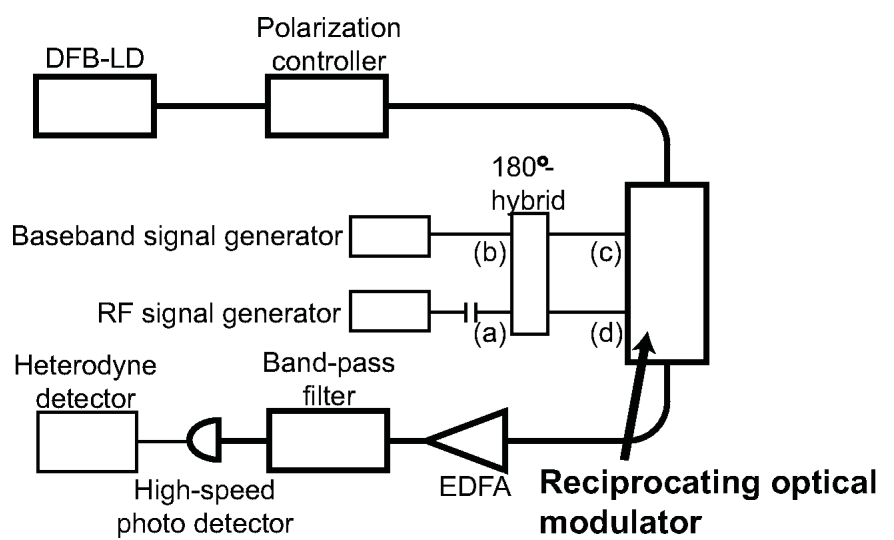

Fig. 2. Experimental setup for amplitude modulation on a ROF signal generated by an ROM. (a) RF signal port, and (b) baseband signal port. The RF signal is fed to the ROM through port (c) and (d), while the baseband signal is applied through port (d).

of (b) that was DC-coupled with the input port of the modulator's electrode (c). This DC port was wideband enough to feed the baseband signal into the ROM without signal distortion. The DC bias had a role for fine adjustment of the effective path length between the FBGs and determines interferometric conditions. The baseband signal was PRBS binary amplitude-shift-keying signal whose bit sequence length was $2^{10}-1$.

The amplitude modulated radio-on-fiber signal was introduced into highspeed photodiode after amplified with an erbium-doped fiber amplifier (EDFA). The signal was converted to amplitude-modulated millimeter-wave signal by the photodetection, and then demodulated by a heterodyne receiver. In the receiver, the full-bandwidth of the IF filter and the half-bandwidth of the baseband filter were $10 \mathrm{MHz}$.

First, millimeter-wave-band ROF signal was generated by the ROM. Harmonic sideband components of 4.4-GHz RF signal came up around the stop band of the FBG. Then, we applied 2-Mbit/s baseband signal on the ROM to modulate the ROF signal. Fig. 3 (a) shows the amplitude-modulated millimeter-wave RF spectrum measured at the output of the photodetector. The center frequency of the millimeter-wave signal was $52.8 \mathrm{GHz}$, which was the 12-th harmonic of the input RF signal. The eye pattern of the demodulated signal using the heterodyne receiver was shown in Fig. 3 (b). Well-opened eye pattern was obtained and effectiveness of the internal modulation scheme was proved.

In this experiment, the DC-bias voltage and the amplitude of the input baseband signal were aligned at the optimal conditions where a modulation depth for amplitude modulation was maximized. The peak-to-peak voltage of the input signal was $2.0 \mathrm{~V}$ which was much less than the half-wave voltage of the phase modulator embedded in the ROM $(6.4 \mathrm{~V})$. The decrease of the voltage is owing to the reciprocating modulation during the recirculation of the lightwaves in the ROM. 
(a)

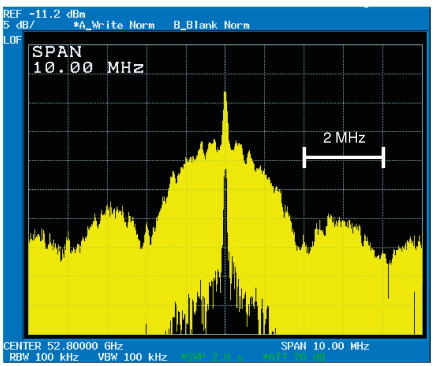

(b)

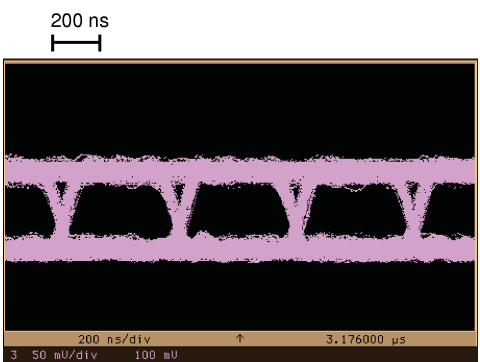

Fig. 3. (a) RF spectrum of generated amplitudemodulated millimeter-wave signal; center frequency: $52.8 \mathrm{GHz}, 1 \mathrm{MHz} / \mathrm{div}$,

(b) Eye-pattern of demodulated signal; $200 \mathrm{~ns} / \mathrm{div}$

\section{Conclusions}

In this paper, we have demonstrated amplitude modulation on millimeterwave signal using a ROM. Amplitude modulated millimeter-wave signal was generated by internal modulation on a DC-bias voltage introduced on the ROM. The required voltage for on-off keying was $2.0 \mathrm{~V}$, which was much less than the $\mathrm{V}_{\pi}$ of typical $\mathrm{LiNbO}_{3}$ modulators.

\section{Acknowledgments}

We would like to express our appreciation to Dr. M. Tsuchiya for fruitful discussions. 